\title{
Carcinoma of gastro-oesophageal junction in a pregnant woman
}

\author{
Katarzyna Kozak, Barbara Łochowska, Józef Kozak, Mariusz Łochowski \\ Department of Thoracic Surgery, Medical University of Lodz, Memorial Copernicus Hospital, Lodz, Poland
}

Gastroenterology Rev 2018; 13 (2): 163-165

DOI: https://doi.org/10.5114/pg.2018.75822

Address for correspondence: Mariusz tochowski MD, PhD, Department of Thoracic Surgery, Medical University of Lodz, Memorial Copernicus Hospital, 62 Pabianicka St, 93-513 Lodz, Poland, phone: + 48602776 887, fax: +48 4268950 10, e-mail: marilo@op.pl

Gastric carcinoma during pregnancy is extremely rare, with reported incidence of $0.016-0.1 \%$. However, the numbers may have increased in recent years because of the increase in maternal age at the time of the first pregnancy $[1,2]$. The diagnosis of gastric cancer in pregnancy is often delayed and it is detected at an advanced stage. No standard treatment has been established because of its rarity, delay in diagnosis, and limited treatment options. A major concern in these cases is the condition of the foetus. There are different views about whether or not to terminate the pregnancy and whether to apply systemic therapy.

In this letter, we present a case of advanced stage cardia carcinoma that was diagnosed in the first trimester of pregnancy.

A 42-year-old woman, gravida 4, para 2 was admitted to our clinic in January 2012 with diagnosis of cardia cancer (adenocarcinoma). The patient denied any comorbidities, and family history was not significant. She had not been treated for gastroesophageal reflux disease or Helicobacter pylori infection in the past. Before the conception, the patient was an active smoker. She denied abusing alcohol. The patient was 17 weeks pregnant on admission. At seventh week of gestation she presented loss of appetite, vomiting, abdominal pain, and dysphagia. At the eighth week upper gastrointestinal endoscopy demonstrated a tumour originating from the cardia (Figure 1). The histopathological diagnosis was gastric carcinoma (adenocarcinoma tubulare). Pregnancy was dated as the eighth week in obstetric ultrasound examination. Development of the foetus was normal. There were no abnormalities other than increased thickness of the cardia wall in an abdominal ultrasound examination. The patient and family were informed about her situation, she did not agree to terminate the pregnancy, and surgery was planned as the treatment of choice. At 18 weeks of gestation a trans hiatal (Orringer) operation was performed. There were no perioperative complications. Histological examination revealed the tumour to be of intestinal type. According to Lauren classification - adenocarcinoma tubulare G3 cardia, $5.5 \times 4.9 \times 3.5 \mathrm{~cm}$ in size, infiltrating entire thickness of the cardia wall and surrounding fat tissue. Immunohistochemical examination detected overexpression of HER 2 receptor. Metastases in 2 lymph nodes was found ( $\mathrm{PT} 3 \mathrm{~N} 1 \mathrm{M} 1$ ). Surgical margin was negative for tumour cells Postoperatively, foetal ultrasonography confirmed the well-being of the foetus. The patient refused to have any systemic therapy applied before the delivery. Caesarean section was performed at 33 weeks and a female newborn was delivered with weight $2200 \mathrm{~g}, 43 \mathrm{~cm}$ in length, and Apgar score $8 / 10$ at 1 and 5 min, respectively. Postpartum she received chemotherapy (capecitabine + cisplatin + trastuzumab). The patient was well for 1 year following the operation, after which dissemination of the disease was found (Figure 2). She received the second-line therapy of epirubicin, oxaliplatin, and capecitabine according to the EOX protocol. Patient died 1.5 years after the neoplasm was diagnosed. The child is now four years old and she is doing well.

The incidence of cancer during pregnancy is approximately 1 per 1000 pregnant women [2]. Pregnancy itself does not cause cancer and pregnant women do not have an increased risk of developing cancer. The most common tumours diagnosed during pregnancy are the same that are diagnosed in females of childbearing age and include: breast cancer, cervical cancer, lymphoma, leukaemia, and lung cancer [1]. Oesophageal and gastric carcinoma are extremely rare [3]. Gastric cancer during pregnancy was first reported by Fujimura and Fukunda in 1916 [4]. The pathogenesis of pregnancyassociated gastric carcinoma remains unknown. The immunosuppressive influence of pregnancy, the pro- 


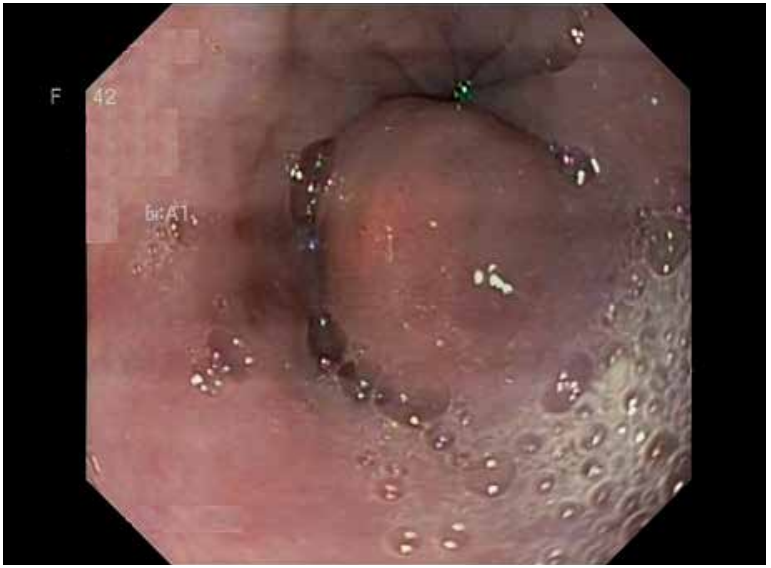

Figure 1. Endoscopy: tumour in gastro-oesophageal junction

moter effect of sex hormones, higher rate infection H. pylori ( $26.6 \%$ vs. $11.6 \%)$, and increased blood circulation during pregnancy are presumed to provoke the rapid growth and spread of cancer [4-6]. Risk factors that were present in most of the cases include: family history of gastric cancer, immunosuppressive disease, drugs, and severe smoking [4]. Currently, whether or not pregnancy accelerates gastric neoplasm is an issue of debate. On the other hand, gastric cancer in pregnancy comparable to young gastric cancer cases is characterised by preponderance to female sex, proximal localisation of the tumour, poor differentiation, more aggressive course of the disease, and poorer prognosis [5]. Advanced tumour stage was found in $92-97 \%$ of the cases [4]. There are several possible reasons for such poor prognosis. Delayed diagnosis is the main reason because up to $80 \%$ of patients are asymptomatic during the early stages of gastric cancer and it is difficult to distinguish between early symptoms of cancer and pregnancy-induced symptoms such as nausea and vomiting. Nausea and vomiting improve by the $16^{\text {th }}$ week of gestation in $90 \%$ of cases and by the $20^{\text {th }}$ week of gestation in $99 \%$ of cases [6]. In our patient dysphagia was the symptom that do not occur in pregnancy and it was an indication for endoscopy in the eighth week of pregnancy. Endoscopic examination has been reported to be safe in pregnancy [4]. The evaluation of gastrointestinal alarm symptoms in pregnant patients outweighs the potential risk. The second reason for delayed stomach cancer detection in young individuals is poorly differentiated adenocarcinomas and reaching and advanced stage [2]. Diffuse microscopic type of gastric cancer represented $75 \%$ of pregnancy-induced gastric cancer cases [2]. In our case patient was 42 years old and she had cancer typical for the older population. No tumour marker (CEA, CA19-9) elevation was observed in gastric cancer during pregnancy [6]. Metastasis of a malignant tumour to the placenta or to the foetus is extremely rare; only 2 cases of metastasis of gastric cancer have been reported [6]. The prognosis of gastric cancer during pregnancy is generally considered as poor. The survival rate for the mother after 1 year is about 20\%, after 2 years $15 \%$, and after 3 years the survival rate is $8 \%$. Foetal survival rate is $70 \%$ and improves with advancing gestational age with rates close to $100 \%$ in foetuses beyond a gestational age of 30 weeks [7]. The treatment options for pregnant women with cancer are the same as those of non-pregnant women with cancer. The type of treatment and time when it may be given depends on the location, type and stage of the cancer, the condition and gestational age
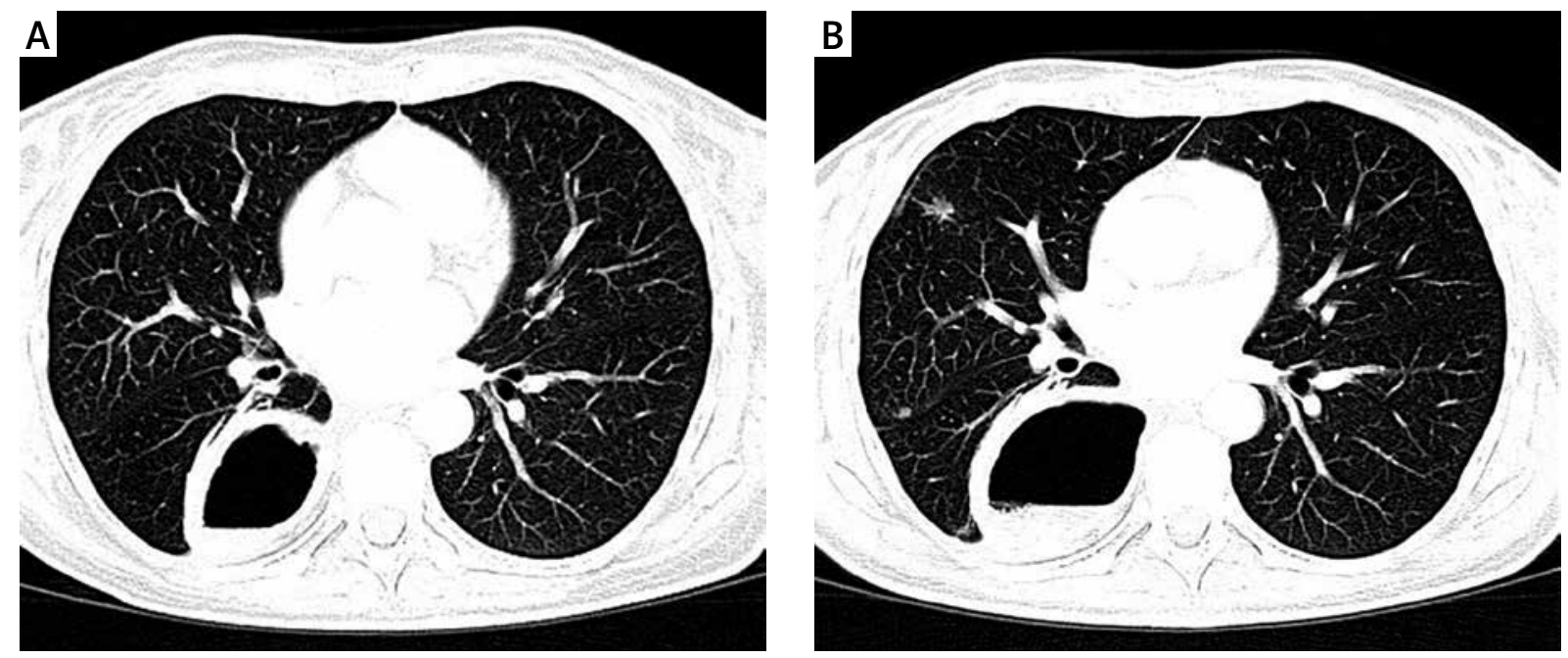

Figure 2. Computed tomography scan. A - After the first line of therapy. B - Dissemination of the disease: pulmonary metastases before the second line of therapy 
of the foetus, and the wishes of the mother [8]. Cancer treatment used during pregnancy may include: surgery, chemotherapy, and sometimes radiation therapy $[1,2$, $5,6]$. Most papers accept Ueo et al.'s proposed diagnostic and treatment algorithm from 1991 [4, 7], in which they briefly recommended the termination of pregnancy in cases until 25 weeks of gestation. Beyond 24 weeks of gestation they recommended resection of the gastric cancer. After week 30, it is suggested that the foetus be delivered followed by radical operation on the gastric cancer. The administration of chemotherapy is possible after first trimester of pregnancy. The first trimester is the period of organogenesis, and chemotherapy can exert a significant teratogenic effect on the child. The risk of malformations is approximately $7-17 \%$ when single treatment is used and up to $25 \%$ in cases of combination therapy [1]. After the first trimester the risk value is $1.3 \%$ and is similar to the general population without chemotherapy treatment [1]. Taking into account that most women do not survive 2 years after delivery and usually the foetus is healthy, we recommend choosing therapeutic options that are considered as better and safer for the foetus, as in our case.

The only possible improvement in the prognosis of gastric cancer in pregnancy is early detection. Upper gastrointestinal endoscopy is advisable in suspicious cases before implementation of treatment with antacid medications. Survival of mother and foetus must be taken into account before making a treatment decision.

\section{Conflict of interest}

The authors declare no conflict of interest.

\section{References}

1. Esposito S, Tenconi R, Preti V, et al. Chemotherapy against cancer during pregnancy. A systematic review on neonatal outcomes. Medicine (Baltimore) 2016; 95: e4899.

2. Kim EY, Jun KH, Ji JH, et al. Laparoscopic gastrectomy followed by chemotherapy for advanced gastric cancer diagnosed during pregnancy: a case report. Anticancer Res 2016; 36: 4813-6.

3. Akhil P, Apurva A, Asha S, et al. Esophageal carcinoma in preg nancy. J Obstet Gynaecol India 2014; 64: 53-4.

4. Unek IT, Celtik A, Alacacioğlu A, et al. Gastric carcinoma during pregnancy: report of a case. Turk J Gastroenterol 2007; 18: 41-3.

5. Cift T, Aydogan B, Akbaş M, et al. Gastric carcinoma diagnosed at the second trimester of pregnancy. Case Rep Obstetrics Gynecol 2011; 2011: 532854.

6. Yoshida M, Matsuda H, Furuya K. Successful treatment of gastric cancer in pregnancy. Taiwanese J Obstet Gynecol 2009; 48: $282-5$.

7. González-Mesa E, Armenteros MA, Molina A, et al. Fulminant evolution of stomach cancer during pregnancy. Austin J Clin Case Rep 2014; 11: 1051.
8. Skrzypczyk-Ostaszewicz A, Rubach M. Gynaecological cancers coexisting with pregnancy - a literature review. Contemp Oncol 2016; 20: 193-8.

Received: 26.12 .2017

Accepted: 19.03 .2018 